7.Gehlsen, K.R., H.N. Wagner, and M.J.C. Hendrix. 1984. Membrane invasion culture system (MICS). Med. Instrum. 18:268-271.

8.Hendrix, M.J.C., E.A. Seftor, R.E.B. Seftor, and I.J. Fidler. 1987. A simple quantitative assay for studying the invasive potential of high and low human metastatic variants. Cancer Lett. 38:137-147.

9.Chu, Y.W., P.C. Yang, S.C. Yang, Y.C. Shyu, M.J.C. Hendrix, R. Wu, and C.W. Wu. 1997. Selection of invasive and metastatic subpopulations from a human lung adenocarcinoma cell line. Am. J. Respir. Cell Mol. Biol. 17:353-360.

10.Shih, J.Y., S.C. Yang, T.M. Hong, A. Yuan, J.J.W. Chen, C.J. Yu, Y.L. Chang, Y.C. Lee, et al. 2001. Collapsin response mediator protein- 1 and the invasion and metastasis of cancer cells. J. Natl. Cancer Inst. 93:1392-1400.

11.Bloomfield, K.L., B.L. Baldwin, D.G. Harkin, and K.F. Tonissen. 2001. Modification of the Boyden chamber to improve uniformity of cell invasion of Matrigel-coated membranes. BioTechniques 31:1242-1246.

12.de la Monte, S.M., S.A. Lahousse, J. Carter, and J.R. Wands. 2002. ATP luminescencebased motility-invasion assay. BioTechniques 33:98-106.

13.Sasaki, C.Y. and A. Passaniti. 1998. Identification of anti-invasive but noncytotoxic chemotherapeutic agents using the tetrazolium dye MTT to quantitate viable cells in Matrigel. BioTechniques 24:1038-1043.

14.Vipra, M.R. and J.M. Chiplonkar. 2002. Vital stain to study cell invasion in modified Boyden chamber assay. BioTechniques 33:1200-1204.

15.Yamakawa, S., Y. Furuyama, and N. Oku. 2000. Development of a simple cell invasion assay system. Biol. Pharm. Bull. 23:12641266.

Received 27 February 2004; accepted 12 July 2004.

Address correspondence to Wun-Shaing Wayne Chang, President's Laboratory, National Health Research Institutes, 128 Yen-Chiu-Yuan Road, Section 2, Taipei 115, Taiwan.e-mail:wayne@nhri.org.tw

\title{
Preparation of chimeric genes without subcloning
}

\author{
Jozica Vasl, Gabriela Panter, Mojca Bencina, and Roman Jerala \\ National Institute of Chemistry, Ljubljana, Slovenia
}

BioTechniques 37:726-730 (November 2004)

\section{INTRODUCTION}

PCR methods are widely used for the modification of proteins, including point mutations, deletions, and insertions, as well as for gene ligation. Subcloning of PCR-generated gene product into the appropriate expression vector often decelerates this process; therefore, methods that avoid the need for subcloning, such as the QuikChange ${ }^{\circledR}$ mutagenesis approach, are much faster $(1,2)$. Chimeric genes represent a very useful tool for the study of protein function. They allow for the addition of different functionalities, such as various labels (e.g., fluorescent, enzymatic activities resulting in luminescence or colored products, and immuno-tags), domains for protein oligomerization, and for combinations of functional fragments of different proteins. Chimeric genes can be produced by engineering appropriate restriction sites between the gene fragments or, alternatively, by gene splicing by extension of the overlap, where the PCR-generated product needs to be subcloned into the vector of choice (3).

Here we describe an approach for the generation of chimeric genes based on back-to-back PCR, without the need for subcloning. The only requirement of this approach is that the original genes are available in the same or in a related vector,

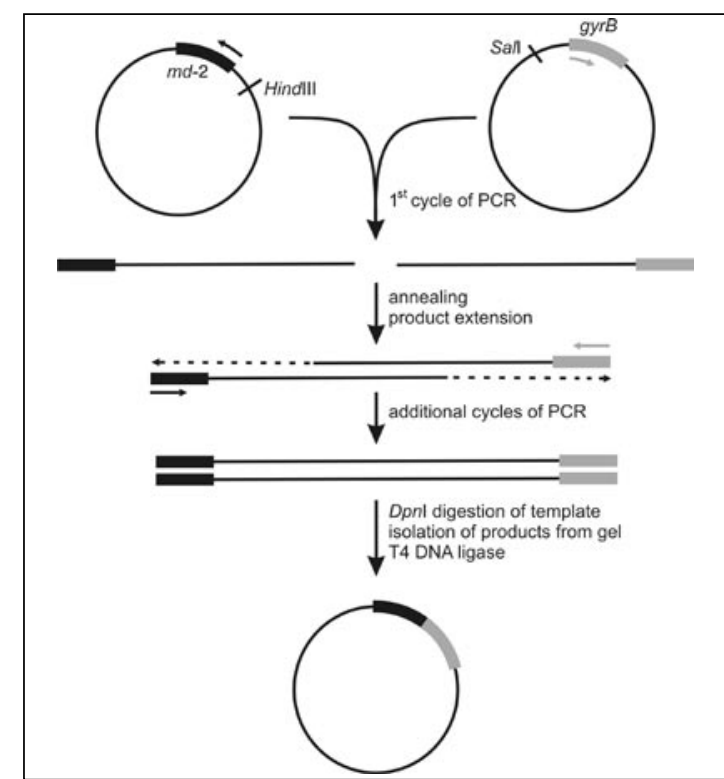

Figure 1. Diagram of the preparation of chimeric genes without subcloning. Both genes to be ligated have to be available in the same type of vector (e.g., expression plasmid) of a pET series. The whole procedure consists of four steps: (i) cleavage of the template plasmids with different enzymes, (ii) PCR, (iii) purification of products with the removal of the remaining template plasmids, and (iv) ligation. Two phosphorylated primers oriented back-to-back are designed, each complementary to the selected region of the genes to be ligated, with the possibility of adding a linker sequence to their $5^{\prime}$ ends. Template plasmids are cut prior to PCR with different enzymes (in this case, HindIII and SalI) $5^{\prime}$ of each primer so that polymerization products of the first PCR cycle contain a long stretch of complementary sequence. In the next PCR cycle, products of the first cycle are annealed, extended to a final length, and amplified in subsequent cycles. Remaining template plasmids originating from bacteria are digested with $D p n \mathrm{I}$, and purified reaction products are circularized by blunt end ligation. 
primer, in the region common to both vector templates. Polymerization from primers in the first PCR extension step results in two single-stranded products based on two different templates. Because each plasmid template is cut with a different enzyme, extension of the primer ends at a different position, providing a long complementary region that is common to the vector in both templates (Figure 1). Singlestranded PCR products are annealed and extended in the next PCR cycle, resulting in a final product that is amplified in the subsequent cycles. The final PCR products are circularized by ligation, which brings together the two extreme ends of the product, corresponding to the ligated gene fragments (Figure 1). For the purpose of ligation, primers have to be phosphorylated or, alternatively, PCR product can be phosphorylated after the amplification and before ligation. As a precaution, we have incorporated additional digestion of the original template plasmid with $D p n I$, which cleaves the methylated template plasmid isolated from bacteria but not the PCR product. Linear PCR product might also be able to transform bacteria at low efficiency, as previously described (4).

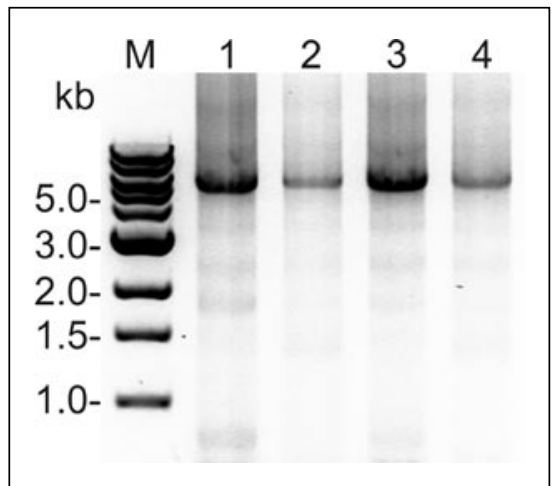

Figure 2. Production of an amplified DNA fragment containing full-length vector with gene fragments from different genes at their ends. The $D p n I$-treated PCR mixture prepared according to the diagram in Figure 1 was purified by $1.2 \%$ agarose gel electrophoresis. Each lane represents the PCR mixture from differently treated templates prior to PCR. M, 1-kb ladder standard (BenchTop 1Kb DNA Ladder; Promega, Madison, WI, USA); lane 1, HindIII and SalI cleaved $m d-2$ and $g y r B$ template plasmid, respectively; lane 2, HindIII cleaved $m d-2$ template and untreated $g y r B$ template plasmid; lane 3 , untreated $m d-2$ template and SalI cleaved gyrB template; lane 4 , both $m d-2$ and $g y r B$ templates plasmid have not been cleaved.
Our goal was the preparation of a fusion protein between human MD-2 and $24 \mathrm{kDa}$ fragment of Escherichia coli gyrase B to investigate the influence of MD-2 dimerization on signal transduction. Gyrase B dimerizes in the presence of double-headed inhibitor coumermycin and is therefore a suitable partner, where dimerization is induced upon the addition of coumermycin (5). Based on structural arguments, we decided to prepare fusion protein of MD-2 with gyrase B domain fused at its $\mathrm{C}$ terminus. We used $m d-2$ cloned in $\mathrm{pET} 14 \mathrm{~b}$ and $g y r B$ cloned in pET3a (Novagen, Dermstadt, Germany) plasmids $(6,7)$, which were isolated with the GenElute ${ }^{\mathrm{TM}}$ Plasmid Miniprep Kit (Sigma, St. Louis, MO, USA). We have designed oligonucleotides 5'-ACCAGAACCATTTGAATTAGGTTGGTGAAGG- ${ }^{\prime}$ and 5'-TCTGGTTCTTCGAATTCTTATGACTCCTCC-3', where the underlined region corresponds to the region complementary to the end of $m d-2$ and to the beginning of $\operatorname{gyr} B$, respectively, while the rest of the oligonucleotide corresponds to the sequence coding for hydrophilic flexible linker SGSSGS. Primers were synthesized by GenScript (Scotch Plains, NJ, USA), phosphorylated at their $5^{\prime}$, and purified by polyacrylamide gel electrophoresis (PAGE). PCR was performed in a $20-\mu \mathrm{L}$ final volume containing 100 ng each of the two plasmids. Plasmid pET14b carrying $m d-2$ and plasmid pET3a carrying gyrB have been previously cleaved with HindIII and SalI (each $10 \mathrm{U}$ ), respectively (Figure 1). Enzymes were heat-inactivated prior to addition to the reaction mixture, which also contained $20 \mathrm{pmol}$ primers, 2.5 $\mathrm{mM}$ dNTP, $1.25 \mathrm{mM} \mathrm{MgCl}{ }_{2}$, and $1 \mathrm{U}$ $T t h$ and $P f u$ DNA polymerase mixture from a CERTAMP Long Amplification Kit (Biotools, Madrid, Spain). Buffer conditions were as recommended by the manufacturer (Biotools). The thermal cycler was programmed for an initial 3-min denaturation at $94^{\circ} \mathrm{C}$, followed by 30 cycles of 1 min denaturation at $94^{\circ} \mathrm{C}, 30 \mathrm{~s}$ annealing at $61^{\circ} \mathrm{C}$, and 10 min elongation at $72^{\circ} \mathrm{C}$, concluding with a final 11-min elongation step at $72^{\circ} \mathrm{C}$. In order to remove any remaining methylated template DNA, the PCR mixture was incubated with
$10 \mathrm{U}$ of $D p n \mathrm{I}$ (New England Biolabs, Beverly, MA, USA) (8), which were added directly to the PCR mixture. After $D p n$ I digestion, the PCR products were fractionated with agarose gel electrophoresis (Figure 2) and gel-purified using a MiniElute ${ }^{\mathrm{TM}} \mathrm{Gel}$ Extraction Kit (Qiagen, Hilden, Germany). One hundred nanograms of each column-purified DNA were further ligated with the Quick Ligation ${ }^{\mathrm{TM}}$ Kit (New England Biolabs) using 1 $\mathrm{U}$ of T4 DNA ligase and transformed into competent $E$. coli $\mathrm{DH}-5 \alpha$ cells with the efficiency of chimeric gene construction between $20 \%$ and $50 \%$, depending on the plasmid cleavage by restriction endonuclease.

It is also possible to cleave only one of the plasmids because the shorter product of polymerization will anneal to the complementary strand and result in the amplified product in the next PCR rounds (Figure 2, lanes 2 and 3). We obtained the correct final product of the correct size even without prior cleavage with restriction enzymes (Figure 2, lane 4). The successful polymerization probably results from the presence of shorter polymerization products, which can anneal to the complementary strand and are amplified in subsequent cycles. As expected, no product was obtained if only one of the plasmid templates was present in the PCR mixture. In this particular example, we have used two partners cloned in similar but not the same vectors (pET3a and pET14b), which are identical over most of the sequence. Therefore, this approach can be used even if the two vectors have only limited common regions (e.g., selection markers) provided that the plasmid is cleaved in this region.

Given the availability of a collection of genes in the same type of vector, which is usually the case in gene libraries, the described method probably enables the fastest generation of chimeric genes because it can be accomplished in a couple of hours. This method allows for the generation of chimeras of different orientation as well as the addition of linker sequences between the genes by the addition of desired extension at $5^{\prime}$ of primers. The number of possible chimeras that can thus be rapidly generated increases as the square of the library size. 


\section{ACKNOWLEDGMENTS}

The work was supported by Ministry of Education, Science and Sport of Slovenia.

\section{COMPETING INTERESTS STATEMENT}

The authors declare no competing interests.

\section{REFERENCES}

1.Wang, W. and B.A. Malcolm. 2002. Twostage polymerase chain reaction protocol allowing introduction of multiple mutations, deletions, and insertions, using QuikChange site-directed mutagenesis. Methods Mol. Biol. 182:37-43.

2.Cabre, A., J. Girona, G. Zalba, M.U. Moreno, J. Diez, and L. Masana. 2004. Generation of eight adjacent mutations in a single step using a site-directed mutagenesis kit. Clin. Chem. Lab. Med. 42:384-386.

3.Mehta, R.K. and J. Singh. 1999. Bridgeoverlap-extension PCR method for constructing chimeric genes. BioTechniques. 26:10821086.

4.Russell, C.B., D.S. Thaler, and F.W Dahlquist. 1989. Chromosomal transformation of Escherichia coli recD strains with linearized plasmids. J. Bacteriol. 171:2609. 2613.

5.Farrar, M.A., S.H. Olson, and R.M. Perlmutter. 2000. Coumermycin-induced dimerization of GyrB-containing fusion proteins. Methods Enzymol. 327:421-429.

6.Plaper, A., M. Golob, I. Hafner, M. Oblak, T. Solmajer, and R. Jerala. 2003. Characterization of quercetin binding site on DNA gyrase. Biochem. Biophys. Res. Commun. 306:530-536.

7.Gruber, A., M. Mancek, H. Wagner, C.J. Kirschning, and R. Jerala. 2004. Structural model of MD-2 and functional role of its basic amino acid clusters involved in cellular LPS recognition. J. Biol. Chem. 279:2847528482.

8.Weiner, M.P., G.L. Costa, W. Schoettlin, J. Cline, E. Mathur, and J.C. Bauer. 1994. Site-directed mutagenesis of double-stranded DNA by the polymerase chain reaction. Gene 151:119-123.

Received 23 June 2004; accepted 13 July 2004.

Address correspondence to Roman Jerala, National Institute of Chemistry, Hajdrihova 19, Ljubljana 1000, Slovenia. e-mail: roman.jerala@ki.si

\title{
High-throughput extraction of arthropod vector and pathogen DNA using bead milling
}

\author{
Christopher J. Allender ${ }^{1}$, W. Ryan Easterday ${ }^{1}$, Matthew N. Van Ert ${ }^{1}$, David \\ M. Wagner ${ }^{1}$, and Paul Keim ${ }^{1,2}$ \\ ${ }^{1}$ Northern Arizona University, Flagstaff and ${ }^{2}$ The Translational Genomics Research \\ Institute (TGen), Phoenix, AZ, USA
}

BioTechniques 37:730-734 (November 2004)

Many important human diseases require an arthropod vector to complete their transmission cycle. These vectors provide an efficient means to track and monitor vector-borne pathogens, including Yersinia pestis (plague) (1-3), West Nile Virus (West Nile encephalitis) (4,5), and Borrellia spp. (Lyme disease) (6). Here we present a rapid DNA extraction protocol to isolate pathogen and vector DNA from vectors. Our example involves fleas (Siphonaptera) and their role as vectors of plague, but the techniques are expected to be applicable to many vector-borne pathogens.

As the most prevalent form of plague transmission is flea-mediated, a common method for determining the presence of the disease in an area involves collecting fleas, extracting DNA from those fleas, and screening the extracts for $Y$. pestis DNA using genetic techniques. Disruption of the flea cuticle is the most time-consuming aspect of this method, as it traditionally involves the use of micropestles and enzymes for cell lysis (7). As a result, for efficiency, fleas are usually pooled together for DNA extraction when using these techniques. In contrast, bead milling allows for rapid, highthroughput disruption of vector cuticles. Although bead-beating techniques have been described previously (8), here we present significant improvements in throughput over these previous techniques. These improvements make it cost- and time-effective to examine individual vectors, as opposed to vector pools, which facilitates analy- sis of detailed disease patterns, such as presence and quantity of pathogens within individual vectors.

Our method for extracting DNA from vectors is novel as it couples automated 96-well bead mill mechanical disruption with a commercially available 96-well DNA purification kit. The mechanical disruption for this protocol uses a 2000 Geno/Grinder mill homogenizer (SPEX CertiPrep, Metuchen, NJ, USA) in conjunction with 2-mm very high-density zirconium oxide beads (Glen Mills, Clifton, NJ, USA). The 96-well DNA purification protocol is the 96-well DNeasy ${ }^{\circledR}$ Tissue Kit (Qiagen, Valencia, CA, USA) modified for use with the QIAvac vacuum filtration system. This system allows for complete processing of 96 samples in about 2 to $3 \mathrm{~h}$, from loading samples to elution of DNA $(100 \mu \mathrm{L})$.

The process begins with adding beads, reagents, and samples into a deep-well 96-microtube plate (Qiagen). First, six beads are added to each 1.2-mL well of the microtube plate using our custom-designed bead transfer plate. The transfer plate was created by drilling 96, 12-mm-deep wells into a $10 \times 14 \times 1.3 \mathrm{~cm}$ piece of clear plexiglass. The wells of this plate are filled with beads by pouring beads across the surface of the plate until each well is full. As the beads are 2 $\mathrm{mm}$ in diameter, only six beads will be contained in each well. The beads are then added to the microtubule plate by placing it upside down on the loading plate and then flipping it over, transferring the beads. Second, $40 \mu \mathrm{L}$ of 